\title{
Prevalence, Predictive Factors and Nomogram of Residual Disease Following Cervical Conization for Adenocarcinoma in Situ
}

\author{
Sukkasame Pichamon, Boonyapipat Sathana
}

Division of Gynecologic Oncology, Department of Obstetrics and Gynecology, Faculty of Medicine, Prince of Songkla University, Songkhla, Thailand.

\begin{abstract}
Objectives: To evaluate the prevalence of residual disease after conization in AIS women, and to develop a nomogram for predicting residual disease in such patients. Methods: Eighty-three cervical adenocarcinoma in situ (AIS) patients were retrospectively reviewed. Patient data concerning: demographic characteristics, colposcopic findings and diagnosis, type of conization, size of pathologic specimens, pathological characteristics, endocervical curettage (ECC) pathology and subsequent procedures, were collected. The rate of residual disease after conization and predictive factors for residual disease in subsequent hysterectomy were analyzed, and a predictive nomogram for residual disease was developed, based on the multivariate analysis results. The statistical significance was set at a p-value of $<0.05$. Results: The prevalence of residual disease in hysterectomy specimens following conization was $31.8 \%$. Five $(5.7 \%)$ women with AIS, who underwent subsequent hysterectomy, were found to have invasive adenocarcinoma. According to the multivariate analysis results, the predictive factors for residual disease were a positive endocervical margin status [OR 22.5 (95\% CI 4.74, 106.79)] and a depth of specimen of $<8 \mathrm{~mm}$ [OR $8.11(95 \%$ CI 1.12, 58.95)]. A nomogram for the prediction of residual disease in AIS women was developed, based on these predictive factors. After bootstrapping 1000 times, the bootstrap-corrected concordance index value for predicting residual disease was 0.852 . Conclusion: The residual disease was found in $31.8 \%$ of hysterectomy specimens after conization for AIS. Residual disease was strongly associated with a positive endocervical margin and a depth of specimen of $<8 \mathrm{~mm}$. This study reports a feasible nomogram, with an acceptable level of accuracy for predicting the individual risk of residual disease; which may be beneficial in proper management decision-making.
\end{abstract}

Keywords: Adenocarcinoma in situ- cervical dysplasia- conization- nomogram- risk factor

Asian Pac J Cancer Care, 6 (3), 317-322

\section{Introduction}

Adenocarcinoma in situ (AIS) of the cervix is an abnormal cervical glandular lesion that is recognized as a precancerous stage of cervical adenocarcinoma. The incidence of AIS is about 1.25 to 1.59 per 100,000 [1-2] women, which is lower than that of squamous intraepithelial abnormalities. Recently, AIS trends have experienced a relative rise compared to the decreasing incidence of squamous cell abnormalities. Postulated explanations include changes in causative HPV subtypes [3], unknown preventing co-factors for AIS [4] and
Submission Date: 02/11/2021 Acceptance Date: 07/22/2021

\footnotetext{
Corresponding Author:

Dr. Boonyapipat Sathana

Division of Gynecologic Oncology, Department of Obstetrics and Gynecology, Faculty of Medicine, Prince of Songkla University, Hat Yai,

Songkhla, 90110, Thailand.

Email: sathana.t@psu.ac.th
} 
to occur in women of a younger age, and women tend to delay childbearing compared to in the past [5]. Hence, preservative surgery is now playing an increasingly important role in management of AIS. However, the risk of residual disease and co-existing adenocarcinoma after conservative treatment procedures should be of concern. The risks of residual intraepithelial lesions and co-excising malignancies must be discussed with the patient before the commencement of conservative treatment [4].

Previous studies have reported rates of residual disease following conization ranging from $6.3 \%$ to $55.5 \%$, depending on marginal status $[1,6-10]$. The risk of residual disease is related to an age of more than 35 years, persistent abnormal pap smear results, positive margins of conization specimens, presence of AIS in endocervical curettage tissue, smaller cone volume and an invisible squamocolumnar junction on colposcopy [8, 10-12]. However, these findings are not consistent $[8,12]$.

This study aimed to determine the prevalence of residual disease after conization in AIS women, and to identify factors that can predict residual disease in patients with AIS undergoing subsequent hysterectomy. To the best of our knowledge, a predictive nomogram for AIS residual disease following conization has not yet been reported. We, therefore, constructed a nomogram to estimate an individual's probability of having residual disease.

\section{Materials and Methods}

This retrospective study reviewed the data of patients diagnosed with AIS having undergone cervical conization; from January, 2004 to December, 2018, at the Department of Obstetrics and Gynecology of Songklanagarind Hospital, Thailand. Both medical records and pathological reports were reviewed. Patients diagnosed with AIS from conization, and undergoing subsequent hysterectomy were included. On the other hand, women diagnosed with coexisting cervical cancer, completed cervical cancer treatment, or those were pregnant were excluded. This study was approved by the Ethics Committee of the Faculty of Medicine, Prince of Songkla University.

Patient characteristics, colposcopic findings and diagnosis, type of conization, margin status, endocervical curettage (ECC) pathology, maximum diameter and depth of pathological specimen, number of areas of disease involved, and residual disease; based on hysterectomy specimens, were reviewed from medical, pathological, and colposcopic records kept in our hospital's computer database as well as documentation from the colposcopy unit.

All patients in this study underwent colposcopy examination, by a gynecologic oncologist or a $2^{\text {nd }}$-year fellow trainee, at the Gynecologic Oncology Unit. The colposcopic findings and diagnoses were recorded in the colposcopy image database. Either immediate therapeutic conization, or cold knife conization was performed, depending on the colposcopist's preference. Loop conization was performed under local anesthesia, using electrosurgical loops varying in size, depending on the geography of the lesion. Cold knife conization was performed under spinal block in the operating room, by a gynecologic oncologist or a fellow trainee. Endocervical curettage was performed immediately after the conization, and specimens were marked at the 3, 6, 9 and 12 o'clock locations.

The cone specimens were prepared and examined by experience pathologists, as per the standard guidelines for handling cone specimens. Each specimen was fixed with $10 \%$ formalin, and the cone margins were marked with indelible ink. Then the specimen size was measured. The specimen was sectioned at 2-3 mm intervals, along the plan of the endocervical canal, starting at the 12 o'clock mark and embedded in paraffin. The section slides were stained with hematoxylin and eosin. The reported microscopic characteristics were divided into 4 quadrants. If the diagnosis was inconclusive, the results were reviewed by an expert pathologist and gynecologic oncologist, in a clinicopathological conference. Residual cervix, obtained from the hysterectomy procedure, was prepared and examined as a cone specimen. Any abnormal squamous or glandular neoplastic cells in the hysterectomy specimens were considered as residual disease.

The cone margin status was classified as endocervical, ectocervical, and deep margin and categorized as either a negative or positive margin. A positive margin was defined as the presence of any abnormal squamous or glandular neoplastic cells. A negative margin was defined as the absence of any neoplastic cells and the presence of normal cervical tissue. A negative margin status was defined as all categorize margins being negative, while a positive margin status was defined as 1 or more of the categorized margins being positive.

The endocervical specimens were classified as negative, positive or inadequate. Negative was defined as the presence of normal endocervical cells and the absence of any neoplastic cells; whereas, positive was defined as the presence of neoplastic cells. Inadequate endocervical tissue for interpretation was considered negative.

The sample size was calculated using the one-proportion formula, under the assumptions that the prevalence of residual disease was 0.14 [8], the precision was 0.1 , the standard normal variate was 1.96 at a $95 \%$ confidence level and the estimated loss of data was $10 \%$. The calculation resulted in a sample size of 52 patients. All data were double entered into Epidata version 3.1, and analyzed using $\mathrm{R}$ software version 13.1. Descriptive data are presented as percentage, mean and median. Clinicopathological characteristics and colposcopic findings were analyzed using univariate and multivariate studies. The categorical data were analyzed by using the chi-square and Fisher's exact tests. The independent predictors were assessed using the logistic regression model, with a p-value of $<0.05$ being considered statistically significant. Finally, a nomogram for predicting residual disease risk was constructed, based on the results of the multivariate analysis, and its internal validation was determined using the bootstrapping method. 


\section{Results}

\section{Baseline characteristics}

During the study period, 88 women were diagnosed with AIS from conization, and from this,eighty-three women who met the inclusion criteria were identified. Their baseline characteristics and data related to their colposcopy and histology of the conization specimens are shown in Table 1.

The mean age was 47.4 years ( $\mathrm{SD} \pm 9.1$ years; range 27 to 70 years), and most of them were of a premenopausal status. The majority (64\%) was asymptomatic, and 24\% had abnormal vaginal bleeding or discharge. AIS was diagnosed by LEEP in $77.1 \%$ of the participants, and by cold knife conization in the remaining $22.9 \%$. Pure AIS was found in $53.0 \%$ of cases; whereas, the other $47.0 \%$ had co-existing squamous abnormality/cervical intraepithelial neoplasia (CIN). The margins were free from disease in 48 women $(57.8 \%)$, while endocervical margin was involved in 27 women (32.5\%).

Residual disease after conization was present in 25 of the 83 women (30.1\%). Five $(5.7 \%)$ women had invasive adenocarcinoma, and all of them had a positive endocervical margin. The other residual diseases identified were 16 of pure AIS (19.3\%), 2 of AIS with coexisting CIN (2.4\%) and 2 of CIN (2.4\%). All of the conization margins, of both women with residual CIN, were negative. Twenty of the $25(80 \%)$ women with residual disease had at least one positive conization margin, and 76\% (19/25) had a 3-4 quadrant AIS involvement.

\section{Factors associated with residual disease}

From the univariate analysis, the identified positive predictors for residual disease in women with AIS, diagnosed by conization, were at an age of $>45$ years, had menopausal status, a depth of specimen $<8 \mathrm{~mm}$, had presence of CIN, a positive margin status, and histologic results of ECC, as shown in Table 1. When all the significant variables obtained from the univariate analysis were entered into the multivariate model, endocervical margin status and depth of cone specimen resulted as the only two significantly, independent predictors for residual disease after conization (adjusted OR 22.5; 95\%CI 4.74, 106.79; $<<0.001$, and adjusted OR 8.11 ; 95\%CI 1.12, 58.95; $\mathrm{p}=0.037$ ); as shown in Table 2 .

\section{Nomogram and validation}

Based on the findings of the multivariate analysis model, the two most powerful predictive factors for residual disease after conization were used to construct the nomogram (Figure 1). The estimated probability of residual disease could be determined by summing the scores on the given scale. The internal validation of the nomogram was analyzed using the 1000-bootstrap method, and the calibration plot for residual disease after conization. Based on a mean absolute error of 0.039 , the agreement between the prediction of the nomogram, and observation or the concordance index (C-index) was 0.852 (95\% CI, 0.83-0.87).
Table 1. Comparisons of Patient Charateristics, Treatment-related Factors and Histology between Two Groups

\begin{tabular}{|c|c|c|c|}
\hline Total & $\begin{array}{l}\text { No residual } \\
\text { disease } \\
\mathrm{n}=58(\%)\end{array}$ & $\begin{array}{c}\text { Residual } \\
\text { disease } \\
\mathrm{n}=25(\%)\end{array}$ & P-value \\
\hline \multicolumn{4}{|l|}{ Patient characteristics } \\
\hline Age (years) & & & 0.042 \\
\hline$<45$ & $32(55.2)$ & $7(28.0)$ & \\
\hline$>45$ & $26(44.8)$ & $18(72.0)$ & \\
\hline Menopausal status & & & 0.026 \\
\hline Premenopause & $44(75.9)$ & $12(48.0)$ & \\
\hline Menopause & $14(24.5)$ & $13(52.0)$ & \\
\hline Parity & & & 1 \\
\hline Nulliparous & $2(3.4)$ & $1(4.0)$ & \\
\hline Parous & $56(96.6)$ & $24(96.0)$ & \\
\hline $\mathrm{HIV}^{*}$ status & & & 1 \\
\hline No & $57(98.3)$ & $25(100)$ & \\
\hline Yes & $1(1.7)$ & $0(0)$ & \\
\hline \multicolumn{4}{|l|}{ Cervical cytology results } \\
\hline Epithelial abnormality & & & 0.174 \\
\hline Normal & $30(51.7)$ & $16(64.0)$ & \\
\hline ASC-US $/$ LSIL ${ }^{\S \S}$ & $2(3.4)$ & $1(4.0)$ & \\
\hline ASC-H $\mathrm{H}^{\mathrm{f}} / \mathrm{HSIL}{ }^{\mathrm{ff}}$ & $25(43.1)$ & $6(24.0)$ & \\
\hline Squamous cell carcinoma & $1(1.7)$ & $2(8.0)$ & \\
\hline Glandular abnormality & & & 0.354 \\
\hline Normal & $27(46.6)$ & $7(28.0)$ & \\
\hline All $\mathrm{AGC}^{\delta}$ & $10(17.2)$ & $4(16.0)$ & \\
\hline $\mathrm{AIS}^{\epsilon}$ & $13(22.4)$ & $9(36.0)$ & \\
\hline Adenocarcinoma & $8(13.8)$ & $5(20.0)$ & \\
\hline \multicolumn{4}{|l|}{ Specimen characteristics } \\
\hline Method of conization & & & 1 \\
\hline $\mathrm{CKC}^{ \pm}$ & $13(22.4)$ & $6(24.0)$ & \\
\hline LEEP $^{ \pm+}$ & $45(77.6)$ & $19(76.0)$ & \\
\hline Depth of specimen (mm) & & & 0.019 \\
\hline$<8$ & $3(5.2)$ & $6(24.0)$ & \\
\hline$>8$ & $55(94.8)$ & $19(76.0)$ & \\
\hline \multicolumn{3}{|c|}{ Maximum diameter of specimen (mm) } & 0.829 \\
\hline$<24$ & $27(46.6)$ & $13(52.0)$ & \\
\hline$>24$ & $31(53.4)$ & $12(48.0)$ & \\
\hline \multicolumn{2}{|c|}{ Co-existing $\mathrm{CIN}^{\epsilon \epsilon}$ in cone specimen } & & 0.042 \\
\hline Pure AIS ${ }^{\epsilon}$ & $26(44.8)$ & $18(72.0)$ & \\
\hline Mixed $\mathrm{CIN}^{\epsilon} / \mathrm{AIS}^{\epsilon}$ & $32(55.2)$ & $7(28.0)$ & \\
\hline Margin status & & & $<0.001$ \\
\hline All margins negative & $43(74.1)$ & $5(20.0)$ & \\
\hline 1 or more positive margins & $15(25.9)$ & $20(80.0)$ & \\
\hline Ectocervical margin & & & 0.058 \\
\hline Negative & $51(87.9)$ & $17(68.0)$ & \\
\hline Positive & $7(12.1)$ & $8(32.0)$ & \\
\hline Endocervical margin & & & $<0.001$ \\
\hline Negative & $50(86.2)$ & $6(24.0)$ & \\
\hline Positive & $8(13.8)$ & $19(76.0)$ & \\
\hline Deep margin & & & 0.045 \\
\hline Negative & $50(86.2)$ & $16(64.0)$ & \\
\hline Positive & $8(13.8)$ & $9(36.0)$ & \\
\hline
\end{tabular}


Continued Table 1.

\begin{tabular}{|c|c|c|c|}
\hline Total & $\begin{array}{l}\text { No residual } \\
\text { disease } \\
\mathrm{n}=58(\%)\end{array}$ & $\begin{array}{c}\text { Residual } \\
\text { disease } \\
\mathrm{n}=25(\%)\end{array}$ & P-value \\
\hline Endocervical curettage $(\mathrm{ECC})^{¥}$ & & & 0.025 \\
\hline Negative & $52(89.7)$ & $17(68.0)$ & \\
\hline Positive & $6(10.3)$ & $8(32.0)$ & \\
\hline \multicolumn{2}{|c|}{ Number of disease involvement quadrants } & & 0.328 \\
\hline $1-2$ quadrants & $22(37.9)$ & $6(24.0)$ & \\
\hline 3-4 quadrants & $36(62.1)$ & $19(76.0)$ & \\
\hline \multicolumn{4}{|l|}{ Colposcopic characteristics } \\
\hline Colposcopic status & & & 1 \\
\hline Adequate & $24(41.4)$ & $10(40.0)$ & \\
\hline Inadequate & $34(58.6)$ & $15(60.0)$ & \\
\hline Colposcopic impression & & & 1 \\
\hline Normal/LGL ${ }^{\mathfrak{f}}$ & $26(44.8)$ & $11(44.0)$ & \\
\hline $\mathrm{HGL}^{\mathrm{ff}} /$ cancer & $32(55.2)$ & $14(56.0)$ & \\
\hline
\end{tabular}

*HIV, Human Immunodeficiency Virus; ${ }^{\S}$ ASC-US, Atypical Squamous Cells of Undetermined Significance; $\$$ LSIL, Low-Grade Squamous Intraepithelial Lesion; ${ }^{\mp} \mathrm{ASC}-\mathrm{H}$, Atypical Squamous Cells; Cannot Exclude High-Grade Squamous Intraepithelial Lesions; 'FFSIL, High-Grade Squamous Intraepithelial Lesion; ${ }^{\delta} \mathrm{AGC}$, Atypical Glandular Cells; ${ }^{\epsilon}$ AIS, Adenocarcinoma in Situ; ${ }^{\epsilon} \mathrm{CIN}$, Cervical Intraepithelial Neoplasia; ${ }^{ \pm} \mathrm{CKC}$, Cold Knife Conization; ${ }^{ \pm} \mathrm{LEEP}$ Loop Electrosurgical Excision Procedure; ${ }^{\mathrm{L}}$ LGL, Low-Grade Lesion; ${ }^{\mathrm{Ef}} \mathrm{HGL}$, High-Grade Lesion; ${ }^{*} \mathrm{ECC}$, Endocervical Curettage

\section{Discussion}

This current study aimed to determine the prevalence of residual disease after cervical conization for AIS, and its predictive factors as well as to construct a nomogram that can be used to estimate individual risk for residual tumors. We found a $30.1 \%$ prevalence rate of residual disease in hysterectomy specimens, which concurs with the findings of previous studies that have reported rates of $31 \%-38 \%[6-10,13]$. Overall, the rate of adenocarcinoma on subsequent hysterectomy, among our AIS women, was $6.02 \%$. The rate of residual disease generally varies depending on margin status. The rate of a positive margin after conization in this study was $30 \%$, which is consistent with rates ranging between $18.2 \%$ and $48.2 \%$ reported in previous studies [11,14]. Salani [6] and Tierney [12] found the risks for concomitant or subsequent adenocarcinoma to be $0.07 \%$ and $2 \%$ in women with negative margins, and $5.2 \%$ and $12 \%$ in women with positive margins, respectively. Our study confirms these findings, as it was found that about $80 \%$ of women with residual disease after hysterectomy had at least one positive side of conization margin.

This present study identified endocervical margin status and depth of conization are independent factors associated with residual disease after conization. Numerous studies have reported the association between endocervical margin status and residual disease. Our study found positive endocervical margins to be strongly associated with residual disease; an 18-fold increase in risk was detected. This result is consistent with those of previous studies, which have reported positive endocervical margins in all women with residual adenocarcinoma, and no residual cancer in women with negative conization margins $[6,8,12]$. This may be explained by the fact that glandular abnormalities likely occur in and extend into the endocervical canal, rather than the ectocervical area.

Our analysis also found that the depth of the conization specimen is a significant risk factor for residual disease. A conization depth of $<8 \mathrm{~mm}$ was found to reduce the risk of residual disease in multivariate analysis. A greater cone depth signifies a better chance to remove glandular lesions located in the cervical canal as well as a decreased risk for residual disease $[5,15]$. Historically, many studies have shown that cold-knife conization(CKC) is associated with a lower rate of positive margins than LEEP, because it produces a greater specimen volume and depth [13-14]. Therefore, CKC has been recommended as a better therapeutic modality of choice for AIS [14]. However, many recent retrospective studies [16] and meta-analyses have shown that the rates of residual disease after conization between CKC and LEEP in AIS women are not significantly different. In line with previous studies, we show that the method of conization does not associate with residual disease risk. Instead, our results provide evidence to underscore the importance of obtaining an adequate cone depth; irrespective of the mode of excision.

Some studies have reported that a positive endocervical curettage (ECC) finding is a risk factor for residual disease, because ECC may help detect endocervical lesions; however, the data in support of this is inconclusive. The overall accuracy of ECC to predict residual or recurrent AIS has been shown to vary between $58 \%$ and $100 \%$ [6]. Costales [8] and Tierney [12] reported a significant increase in the rate of residual AIS among women with a positive ECC for $60 \%$ and 95\%, respectively. In contrast, Kietpeerakool [7] did not support these findings. They reported that up to $40 \%$ of women with a normal ECC result had residual disease in subsequent hysterectomy specimens, while $50 \%-57 \%$ of women with a positive ECC, regardless of margin status, had no residual disease [7,12-13]. A low sensitivity of $50 \%$ of ECC to detect AIS was also noted in another report [8], which proposed that the invagination of AIS within the cervical stroma may make it difficult to obtain by the curettage procedure [7]. The results of this study confirm the lack of association between ECC status and residual disease. While the extent of the benefits of ECC remains unclear, ECC results should be considered to account for other risk factors in further treatment management.

AIS coexisting with CIN in this study was found in about half of AIS women. This is a similar finding to the prevalence rates ranging from $40 \%$ to $60 \%$ reported by other studies $[5,6,12,13,16,17]$. Coexisting CIN showed a protective association with residual disease; however, it did not result in being a significant, independent factor in the multivariate analysis. Patients with pure AIS were higher risk of persistent disease than those with coexisting CIN $[18,19]$. An explanation for these findings may be that the presence of concurrent $\mathrm{CIN}$ leads to earlier diagnosis and treatment [18]. Bekker [20] identified the 25-genotype HPVs in CIN and AIS lesions, and demonstrated that pure 
Table 2. Multivariate Analysis of Predictive Factors for Residual Disease after Conization in AIS Women

\begin{tabular}{|c|c|c|c|}
\hline & Crude OR $(95 \% \mathrm{CI})$ & Adjusted OR (95\%CI) & P-value \\
\hline \multicolumn{4}{|l|}{ Endocervical margin } \\
\hline Positive vs. negative & $19.79(6.06,64.6)$ & $22.5(4.74,106.79)$ & $<0.001$ \\
\hline \multicolumn{4}{|l|}{ Depth of specimen (mm) } \\
\hline$<8$ vs. $>8$ & $5.79(1.32,25.45)$ & $8.11(1.12,58.95)$ & 0.037 \\
\hline \multicolumn{4}{|l|}{ Coexisting CIN in cone specimen } \\
\hline Mixed CIN/AIS vs. pure AIS & $0.32(0.11,0.87)$ & $0.61(0.15,2.53)$ & 0.499 \\
\hline \multicolumn{4}{|l|}{ Age (years) } \\
\hline$>45$ vs. $<45$ & $3.16(1.15,8.73)$ & $1.44(0.37,5.63)$ & 0.602 \\
\hline \multicolumn{4}{|l|}{ Deep margin status } \\
\hline Positive vs. negative & $3.52(1.16,10.63)$ & $0.68(0.12,3.67)$ & 0.649 \\
\hline \multicolumn{4}{|l|}{ Endocervical curettage } \\
\hline Positive vs. negative & $4.08(1.24,13.43)$ & $1.45(0.25,8.54)$ & 0.68 \\
\hline
\end{tabular}

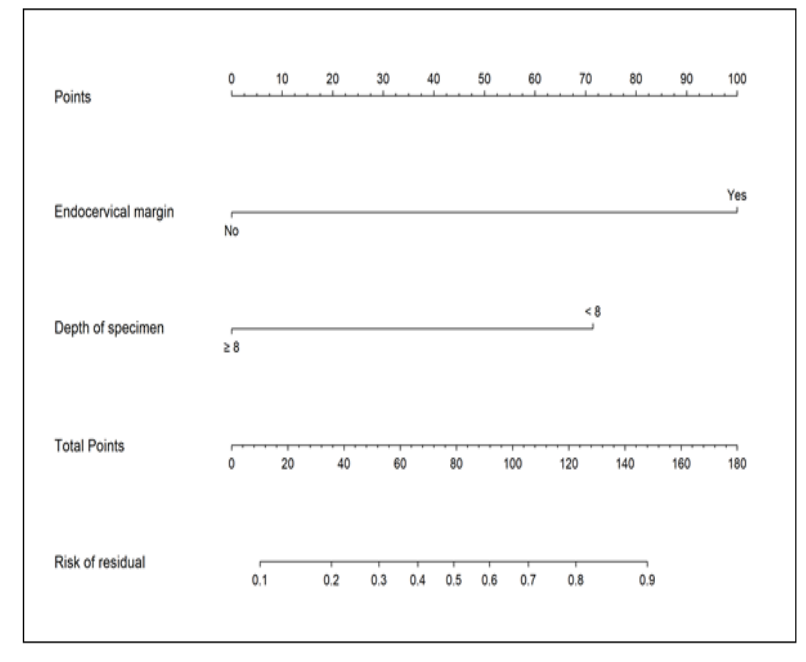

Figure 1. Nomogram to Predict the Probability of Residual Disease after Conization in AIS Women

AIS had a higher rate of the hr-HPV-negative genotype than AIS coexisting with CIN. Variation of HPV biology may also lead to differences in early detection of AIS.

Besides, based on the geographic characteristics of the conization specimen, there have also been reports on histopathologic features and HPV status being predicting factors for residual disease. ElMasri [9] reported that predictive factors for AIS associated with an occult adenocarcinoma were involvement of the endocervical gland by AIS, and the presence of histopathologic features suggestive but not definitive for invasion or the so-called "suspicious of invasion". The presence of both features carried an $18 \%$ chance of having invasive adenocarcinoma in the hysterectomy specimen; this risk increased to $33 \%$ when combined with a positive ECC result. Although, the term "suspicious invasion" is very familiar to pathologists, it requires further validation [9]. HPV DNA positivity has also been reported to be a predictive factor for residual disease in previous studies [21]. However, neither histopathological characteristics, nor HPV status were evaluated in our series; due to lacking data.

The proposed nomogram is a simple mathematical tool, presenting an alignment chart derived from the best multivariate analysis model. This study reports the predictive nomogram attempting to evaluate the risk for residual disease after conization in AIS women. The probability of residual disease was evaluated based on the characteristics of an individual's conization specimen. The $\mathrm{C}$-index value of this nomogram was 0.852 , which indicates that the model has an acceptable level of accuracy. Moreover, it relies on practical or feasible pathological variables. The nomogram provides additional information for quantitative risk predictions for residual disease, before making decisions regarding fertility-sparing management, and the need for a repeat conization before proceeding hysterectomy in women who have completed childbearing.

The strengths of this study lie in the facts that a hysterectomy specimen was used as a gold standard for all subjects. Additionally, predictive factors were scrutinized using a multivariate analysis. On the other hand, the apparent limitations of this study involve information and systematic bias, as a result of its retrospective nature. Some potential risk factors; such as histopathologic features and HPV status, were not included in our analysis. Lastly, based on a limited sample size from a single tertiary center, only two independent predictive factors were identified, and used to construct the predictive nomogram. We recommend that the external validity of this nomogram be evaluated in different cohorts, so as to ensure both its validity and generalizability.

In conclusion, about one third of the participating women had residual disease after conization for AIS. This study has developed a predictive nomogram, which incorporates feasible clinical factors to predict residual disease, with acceptable accuracy. This nomogram provides disease-related risk estimation before conservative treatment decisions are made, and facilitates appropriate follow-up protocols. 


\section{Acknowledgements}

The authors would like to thank all the support givens from the Faculty of Medicine, Prince of Songkhla University. As well as particular thanks to Ms Jirawan Jayupan, from the Epidemiology Unit for her help in data analysis. The authors declare no conflicts of interest.

\section{References}

1. Polterauer S, Reinthaller A, Horvat R, Joura E, Grimm C. Cervical Adenocarcinoma in Situ: Update and Management. Curr Obstet Gynecol Rep. 2013;2:86-93.

2. van der Horst J, Siebers AG, Bulten J, Massuger LF, de Kok IM. Increasing incidence of invasive and in situ cervical adenocarcinoma in the Netherlands during 2004-2013. Cancer Medicine. 201701 19;6(2):416-423. https://doi. org/10.1002/cam4.971

3. Bray F. Incidence Trends of Adenocarcinoma of the Cervix in 13 European Countries. Cancer Epidemiology Biomarkers \& Prevention. 200509 01;14(9):2191-2199. https://doi. org/10.1158/1055-9965.epi-05-0231

4. Massad LS, Einstein MH, Huh WK, Katki HA, Kinney WK, Schiffman M, Solomon D, Wentzensen N, Lawson HW. 2012 Updated Consensus Guidelines for the Management of Abnormal Cervical Cancer Screening Tests and Cancer Precursors. Journal of Lower Genital Tract Disease. 2013 04;17(Supplement 1):S1-S27. https://doi.org/10.1097/ lgt.0b013e318287d329

5. Bai H, Liu J, Wang Q, Feng Y, Lou T, Wang S, Wang Y, Jin $\mathrm{M}$, Zhang Z. Oncological and reproductive outcomes of adenocarcinoma in situ of the cervix managed with the loop electrosurgical excision procedure. BMC Cancer. 201804 24;18(1). https://doi.org/10.1186/s12885-018-4386-6

6. Salani R, Puri I, Bristow RE. Adenocarcinoma in situ of the uterine cervix: a metaanalysis of 1278 patients evaluating the predictive value of conization margin status. American Journal of Obstetrics and Gynecology. 2009 02;200(2):182. e1-182.e5. https://doi.org/10.1016/j.ajog.2008.09.012

7. Kietpeerakool C, Khunamornpong S, Srisomboon J, Kasunan A, Sribanditmongkol N, Siriaungkul S. Predictive value of negative cone margin status for risk of residual disease among women with cervical adenocarcinoma in situ. International Journal of Gynecology \& Obstetrics. 201208 29;119(3):266269. https://doi.org/10.1016/j.ijgo.2012.06.013

8. Costales AB, Milbourne AM, Rhodes HE, Munsell MF, Wallbillich JJ, Brown J, Frumovitz M, Ramondetta LM, Schmeler KM. Risk of residual disease and invasive carcinoma in women treated for adenocarcinoma in situ of the cervix. Gynecologic Oncology. 2013 06;129(3):513-516. https://doi.org/10.1016/j.ygyno.2013.03.015

9. ElMasri W, Walts A, Chiang A, Walsh C. Predictors of invasive adenocarcinoma after conization for cervical adenocarcinoma in situ. Gynecologic Oncology. 2012 06;125(3):589-593. https://doi.org/10.1016/j.ygyno.2012.03.005

10. Kim J, Park J, Kim D, Kim Y, Kim Y, Nam J. The role of loop electrosurgical excisional procedure in the management of adenocarcinoma in situ of the uterine cervix. European Journal of Obstetrics \& Gynecology and Reproductive Biology. 2009 07;145(1):100-103. https://doi.org/10.1016/j. ejogrb.2009.04.011

11. Costa S, Venturoli S, Negri G, Sideri M, Preti M, Pesaresi M, Falasca A, Barbieri D, Zerbini M, Santini D, Sandri MT, Ghiringhello B, Caroppo Venturini N, Syrjänen S, Syrjänen $\mathrm{K}$. Factors predicting the outcome of conservatively treated adenocarcinoma in situ of the uterine cervix: An analysis of
166 cases. Gynecologic Oncology. 2012 03;124(3):490-495. https://doi.org/10.1016/j.ygyno.2011.11.039

12. Tierney K, Lin P, Amezcua C, Matsuo K, Ye W, Felix J, et al. Cervical conization of adenocarcinoma in situ: a predicting model of residual disease. Am J Obstet Gynecol $2014 ; 210: 366$.e1-5.

13. Srisomboon J, Kietpeerakool C, Suprasert P, et al. Factors affecting residual lesion in women with cervical adenocarcinoma in situ after cone excisional biopsy. Asian Pac J Cancer Prev. 2007;8:225-8.

14. Kim M, Hahn H, Lim K, Lee K, Kim H, Hong S, Kim T. The safety of conization in the management of adenocarcinomain situof the uterine cervix. Journal of Gynecologic Oncology. 2011;22(1):25. https://doi.org/10.3802/jgo.2011.22.1.25

15. Salcedo MP, Costales A, Munsell MF, Ramalingam P, Reis RD, Milbourne A, Beitune PE, Schmeler KM. Adenocarcinoma in situ of the Cervix - is Loop Electrosurgical Excision Procedure an Acceptable Alternative to Cold Knife Cone Biopsy?. European Oncology \& Haematology. 2017;13(01):24. https://doi.org/10.17925/eoh.2017.13.01.24

16. Latif NA, Neubauer NL, Helenowski IB, Lurain JR. Management of Adenocarcinoma In Situ of the Uterine Cervix. Journal of Lower Genital Tract Disease. 2015 04;19(2):97102. https://doi.org/10.1097/lgt.0000000000000055

17. Kietpeerakool C, Srisomboon J, Prompittayarat W, et al. Can adenocarcinoma in situ of the uterine cervix be predicted before cervical conization?. Asian Pac J Cancer Prev. 2006;7:522-4.

18. Codde E, Munro A, Stewart C, Spilsbury K, Bowen S, Codde J, Steel N, Leung Y, Tan J, Salfinger S, Mohan G, Cohen P. Risk of persistent or recurrent cervical neoplasia in patients with 'pure' adenocarcinoma-in-situ (AIS) or mixed AIS and high-grade cervical squamous neoplasia (cervical intra-epithelial neoplasia grades 2 and 3 (CIN 2/3)): a population-based study. BJOG: An International Journal of Obstetrics \& Gynaecology. 201708 02;125(1):74-79. https://doi.org/10.1111/1471-0528.14808

19. Song T, Lee Y, Choi CH, Kim T, Lee J, Bae D, Kim B. The effect of coexisting squamous cell lesions on prognosis in patients with cervical adenocarcinoma in situ. European Journal of Obstetrics \& Gynecology and Reproductive Biology. 2015 07;190:26-30. https://doi.org/10.1016/j. ejogrb.2015.04.005

20. Bekkers RLM, Bulten J, Wiersma-van Tilburg A, Mravunac M, Schijf CPT, Massuger LFAG, Quint WGV, Melchers WJG. Coexisting high-grade glandular and squamous cervical lesions and human papillomavirus infections. British Journal of Cancer. 200308 26;89(5):886-890. https://doi. org/10.1038/sj.bjc. 6601204

21. Chen J, Wang Z, Wang Z, Yang X. The risk factors of residual lesions and recurrence of the high-grade cervical intraepithelial lesions (HSIL) patients with positive-margin after conization. Medicine. 2018 Oct;97(41):e12792. https:// doi.org/10.1097/md.0000000000012792

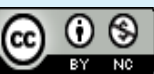

This work is licensed under a Creative Commons AttributionNon Commercial 4.0 International License. 